\title{
The geometric average of curl-free fields in periodic geometries
}

\author{
Klaas Hendrik Poelstra, Ben Schweizer, \\ Maik Urban
}




\title{
The geometric average of curl-free fields in periodic geometries
}

\author{
K. H. Poelstra, B. Schweizer, M. Urban*
}

May 31, 2019

\begin{abstract}
In periodic homogenization problems, one considers a sequence $\left(u^{\eta}\right)_{\eta}$ of solutions to periodic problems and derives a homogenized equation for an effective quantity $\hat{u}$. In many applications, $\hat{u}$ is the weak limit of $\left(u^{\eta}\right)_{\eta}$, but in some applications $\hat{u}$ must be defined differently. In the homogenization of Maxwell's equations in periodic media, the effective magnetic field is given by the geometric average of the twoscale limit. The notion of a geometric average has been introduced by Bouchitte and Bourel in [3]; it associates to a curl-free field $Y \backslash \bar{\Sigma} \rightarrow \mathbb{R}^{3}$, where $Y$ is the periodicity cell and $\Sigma$ an inclusion, a vector in $\mathbb{R}^{3}$. In this article, we extend previous definitions to more general inclusions. The physical relevance of the geometric average is supported by various results, e.g., a convergence property of tangential traces.
\end{abstract}

MSC: 35B27, 78M40, 35B34

Keywords: periodic homogenization; Maxwell's equations

\section{Introduction}

This work provides and analyzes a new definition of the geometric average, a concept that has been introduced in [3] and that turned out to be crucial in the periodic homogenization of time-harmonic Maxwell's equations. We use the setting of periodic homogenization in three dimensions with a periodicity cell $Y=[0,1)_{\sharp}^{3}=\mathbb{R}^{3} / \mathbb{Z}^{3}$ and inclusions $\Sigma \subset Y$ with complement $\Sigma^{*}:=Y \backslash \bar{\Sigma}$. Given a curl-free field $u: \Sigma^{*} \rightarrow \mathbb{R}^{3}$, our aim is to define a vector $\oint_{\Sigma^{*}} u \in \mathbb{R}^{3}$ such that the formula

$$
\int_{\Sigma^{*}} u \wedge \phi=\left(\oint_{\Sigma^{*}} u\right) \wedge\left(\int_{\Sigma^{*}} \phi\right)
$$

holds for a class of test vector fields $\phi$.

In time-harmonic Maxwell's equations with perfectly conducting inclusions, the magnetic field $u^{\eta}$ is defined on a perforated domain $\Omega_{\eta}^{*}$, which is constructed by removing $\eta$-scaled copies of $\Sigma$ from an underlying domain $\Omega$. The magnetic field $u^{\eta}: \Omega_{\eta}^{*} \rightarrow \mathbb{C}^{3}$

\footnotetext{
${ }^{*}$ Technische Universität Dortmund, Fakultät für Mathematik, Vogelpothsweg 87, D-44227 Dortmund, Germany.
} 
satisfies curl $u^{\eta}=w^{\eta}$ for some field $w^{\eta}$ and one can assume that both $\left(u^{\eta}\right)_{\eta}$ and $\left(w^{\eta}\right)_{\eta}$ are bounded in $L^{2}\left(\Omega_{\eta}^{*}\right)$. Under certain assumptions on $\Sigma$, the homogenization yields an effective equation of the form curl $\hat{u}=\varepsilon^{\text {eff }} w$, where $\varepsilon^{\text {eff }}$ is an effective coefficient matrix and $w$ is the weak $L^{2}$-limit of $\left(w^{\eta}\right)_{\eta}$. The important aspect of the effective equation curl $\hat{u}=\varepsilon^{\text {eff }} w$ is that the field $\hat{u}$ is not the weak limit of the sequence $\left(u^{\eta}\right)_{\eta}$, but the geometric limit: Up to subsequences, $\left(u^{\eta}\right)_{\eta}$ converges in the sense of two-scale convergence (as defined in [1]) to a limit $u_{0}=u_{0}(x, y)$, and the field $\hat{u}(x)$ is the geometric average of $u_{0}(x, \cdot)$.

While the homogenization of Maxwell's equations (with split ring inclusions) was performed in [6] without the notion of a geometric average, the notion was introduced in [3] and made the analysis more accessible. The geometric average was introduced in [3] for inclusions $\Sigma$ that are simply-connected and compactly contained in the periodicity cell $(0,1)^{3}$. The authors used a formula similar to (1.1), but in their definition they used scalar products instead of wedge products (and a different class of test functions $\phi$ ). They note, however, that (1.1) is a consequence of their definition. We emphasize that the equivalence of the two definitions holds only for a certain class of geometries.

In order to analyze split ring geometries for perfectly conducting inclusions, the concept of a geometric average was extended in [18] to cover the case of compactly contained inclusions without the assumption of simple connectedness. The concept of a geometric average was extended further in [22] in order to cover the case that the inclusions touch the boundary of the periodicity cell $(0,1)^{3}$.

In the work at hand, we introduce a very general concept of a geometric average encompassing all previous definitions. It turns out to be a natural concept in terms of elementary de Rham theory. We derive properties of the new geometric average and show, in particular, that it allows the homogenization of Maxwell's equations for very general microstructures. Moreover, we derive a result on the convergence of tangential traces: If $\left(u^{\eta}\right)_{\eta}$ is an appropriate sequence and $\hat{u}$ is defined as described above (with the geometric average), then, at the interface $\left\{x_{1}=0\right\}$, there holds

$$
\int_{\left\{x_{1}=0\right\}} \theta u_{3}^{\eta} \rightarrow \int_{\left\{x_{1}=0\right\}} \theta \hat{u}_{3}
$$

as $\eta \rightarrow 0$ for all test functions $\theta$. This result supports the physical relevance of the notion of the geometric average. We have to accept that (1.2) is only conditionally valid; it requires that the microstructure allows for a sufficiently large class of solutions to cell problems.

Literature. The analysis of homogenization problems in domains with periodically distributed holes has a long history, we mention [11] for some typical early results; the approach was considerably simplified with the notion of two-scale convergence in $[1,14]$. While in such standard cases the limit functions are directly linked to the weak limit of the solution sequence as in $[8,10,14]$, this can fail in problems that involve one of the following: (a) high contrast as in [3, 6, 7], (b) Maxwell's equations with perfect conductors and singular geometries [18, 22], (c) Helmholtz problems with singular geometries [17].

The subject of singular homogenization of Maxwell's equations has drawn great interest due to the works $[20,21,23]$, in which artificial materials were introduced that have effectively (i.e., after homogenization) a negative index. Investigations from an applied perspective have been provided in $[12,19]$. One ingredient for the negative index effect can 
be achieved with wires: The wires are constructed as a periodic material, but the inclusion connects opposite points in the periodicity cell. As a result, the length of the single wire in the heterogeneous medium is of order 1 and special homogenization effects can be observed, see $[5,2,13]$. Mie resonances and singular geometries can lead to artificial magnetism, see (a) and (b) above. The combination of wires and bulk resonance can produce a negative index material, see [16].

It was already observed in [15] for the Maxwell equations that physical averaged quantities should use line integrals since the fields $H$ and $E$ are naturally interpreted as 1-forms. For the analysis in an effectively two-dimensional geometry, we mention [9].

Degeneracy issues. For general microstructures $\Sigma \subset Y$, the fundamental equation (1.1) does not determine the vector $\oint_{\Sigma^{*}} u \in \mathbb{R}^{3}$. The admissible test vector fields $\phi: Y \rightarrow \mathbb{R}^{3}$ for (1.1) are curl-free in $Y$ and vanish in $\Sigma$. Relation (1.1) is solvable in the sense that we always find a vector $b \in \mathbb{R}^{3}$ such that $\int_{\Sigma^{*}} u \wedge \phi=b \wedge \int_{\Sigma^{*}} \phi$ holds for all $\phi$. On the other hand, if the space $A\left(\Sigma^{*}\right)=\left\{\int_{\Sigma^{*}} \phi \mid \phi\right.$ admissible $\} \subset \mathbb{R}^{3}$ has a dimension smaller than 2 , the vector $b$ is not uniquely determined. In order to have a unique solution $b$, we introduce a certain subspace $A\left(\Sigma^{*}\right)^{\wedge \perp} \subset \mathbb{R}^{3}$ (see (2.4)) and search for $b \in A\left(\Sigma^{*}\right)^{\wedge \perp}$.

Let us provide here an intuitive description of the spaces $A\left(\Sigma^{*}\right)$ and $A\left(\Sigma^{*}\right)^{\wedge} \downarrow$. We consider the directions $P \subset \mathbb{R}^{3}$ that can be realized with curves in $\Sigma$. More precisely, we study the set $P_{0}:=\left\{\gamma(1)-\gamma(0) \mid \gamma \in C^{0}\left([0,1] ; \mathbb{R}^{3}\right)\right.$ with $\gamma(1)-\gamma(0) \in \mathbb{Z}^{3}$ and $\gamma(t) \in$ $\Sigma+\mathbb{Z}^{3}$ for every $\left.t \in[0,1]\right\} \subset \mathbb{Z}^{3}$ and $P:=\operatorname{span}_{\mathbb{R}} P_{0} \subset \mathbb{R}^{3}$. Then

$$
A\left(\Sigma^{*}\right)=P^{\perp}, \quad A\left(\Sigma^{*}\right)^{\wedge \perp}:= \begin{cases}\mathbb{R}^{3} & \text { if } \operatorname{dim} P \leq 1 \\ P & \text { if } \operatorname{dim} P=2 \\ \{0\} & \text { if } \operatorname{dim} P=3 .\end{cases}
$$

The two subspaces $A\left(\Sigma^{*}\right)$ and $A\left(\Sigma^{*}\right)^{\wedge \perp}$ are not artificially invented for the definition of the geometric average. For example, in the homogenization of Maxwell's equations, certain components of the limiting fields necessarily vanish and, accordingly, certain parts of the equations should not be imposed.

In another form, the subspaces $A\left(\Sigma^{*}\right)$ and $A\left(\Sigma^{*}\right)^{\wedge \downarrow}$ appear in our result on limits of traces; see (1.2). The convergence of any tangential component only holds if a perpendicular tangential vector is contained in $A\left(\Sigma^{*}\right)$.

The above description of the space $A\left(\Sigma^{*}\right)$ indicates that the notion of geometric average depends on topological properties of $\Sigma$. While the description in terms of paths is quite intuitive, it turns out that the description is simplified if we use cohomology instead of homotopy. This is in accordance with the formulation of Maxwell's equations in terms of differential forms leading to de Rham cohomology.

Outline of the paper. After defining the geometric average in Section 2.1, we show how to compute it in a few simple cases in Section 2.2. In particular, we see that in the absence of holes $(\Sigma=\emptyset)$, geometric average and volume average coincide; that the geometric average of a gradient vanishes; and that the geometric average of a constant vector is equal to the orthogonal projection of this constant vector onto $A\left(\Sigma^{*}\right)^{\wedge} \downarrow$. Furthermore, we prove that the geometric average is invariant under deformations of $\Sigma$ that do not affect the topology of the pair $(Y, \Sigma)$. In Section 2.3, we discuss the connection between our definition of the geometric average and previous definitions. 
We begin Section 3 by outlining a macroscopic geometry, also allowing different microstructures in different patches of the domain. We then present a key computation in Theorem 3.1, which shows that curl $u^{\eta}=w^{\eta}$ in $\Omega_{\eta}^{*}$ implies curl $\hat{u} \cdot \int_{Y} \phi=\int_{Y} w_{0} \cdot \phi$ for all test functions $\phi$, where $w_{0}(x, y)$ is the two-scale limit of $w^{\eta}(x)$. This implies that certain components of curl $\hat{u}$ are $\mathrm{L}^{2}$-functions, and therefore that certain components of the tangential trace of $\hat{u}$ exist - namely, the components $\nu \wedge v$, where $\nu$ is a normal vector and $v \in A\left(\Sigma^{*}\right)$. This also holds where two patches with different microstructure meet (see Corollary 3.2).

In Section 4, we use elementary concepts from de Rham theory in order to restate our definition of the geometric average. The geometric average then becomes a map from the first de Rham cohomology group of $\Sigma^{*}$ to a subspace of the first de Rham cohomology group of $Y$,

$$
\oint_{\Sigma^{*}}: \mathrm{H}_{\mathrm{dR}}^{1}\left(\Sigma^{*}\right) \rightarrow A\left(\Sigma^{*}\right)^{\wedge \perp} \subset \mathrm{H}_{\mathrm{dR}}^{1}(Y) .
$$

Using the embedding $i: \mathrm{H}_{\mathrm{dR}}^{1}(Y, \Sigma) \rightarrow \mathrm{H}_{\mathrm{dR}}^{1}(Y)$, the defining relation of the geometric average then becomes

$$
[u \wedge \phi]=\left(\oint_{\Sigma^{*}}[u]\right) \wedge i[\phi], \quad[u] \in \mathrm{H}_{\mathrm{dR}}^{1}\left(\Sigma^{*}\right), \quad[\phi] \in \mathrm{H}_{\mathrm{dR}}^{1}(Y, \Sigma) .
$$

The key idea is that on both the space of vector fields $u$ and the space of test vector fields $\phi$, an equivalence relation can be defined, which leads to the de Rham cohomogy group $\mathrm{H}_{\mathrm{dR}}^{1}\left(\Sigma^{*}\right)$ and the relative de Rham cohomology group $\mathrm{H}_{\mathrm{dR}}^{1}(\Sigma, Y)$, respectively.

These cohomology groups are closely related to the solution space of the cell problems of Maxwell's equations. Indeed, the solutions of the cell problem for the $H$-field yield a set of representatives for $\mathrm{H}_{\mathrm{dR}}^{1}(Y)$. Similarly, the solutions to the cell problem for the $E$-field yield a set of representatives for $\mathrm{H}_{\mathrm{dR}}^{1}(Y, \Sigma)$; see Lemma 4.4 and Lemma 4.5.

In Section 5, we show that under certain assumptions the geometric limit $\hat{u}(x)$ captures all the information which the full two-scale limit $u_{0}(x, y)$ provides. Combined with our previous efforts, this allows us to easily homogenize the time harmonic Maxwell equations in a periodic medium with perfectly conducting inclusions.

\section{The geometric average}

Let $Y$ denote the flat 3 -torus, which can be pictured as the unit cube $[0,1]^{3} \subset \mathbb{R}^{3}$ with opposite faces identified. Occasionally, we will identify it (as a set) with $[0,1)^{3} \subset \mathbb{R}^{3}$. We remark that $\mathrm{H}^{1}(Y)$ is not equal to $\mathrm{H}^{1}\left((0,1)^{3}\right)$. However, it can be identified with the subspace of $\mathrm{H}^{1}\left((0,1)^{3}\right)$ consisting of those functions for which the traces at opposite faces coincide. Similar remarks apply when we consider subsets of $Y$.

We consider a (possibly empty) subset $\Sigma \subset Y$ of the torus which is open and has a Lipschitz boundary. The complementary open set is denoted by $\Sigma^{*}:=Y \backslash \Sigma$. We assume both $\Sigma$ and $\Sigma^{*}$ to be connected. A subset $\Sigma \subset Y$ as above is called an admissible microstructure.

\subsection{Definition of the geometric average}

The space of vector fields for which we define the geometric average is

$$
X\left(\Sigma^{*}\right):=\left\{u \in \mathrm{L}^{2}\left(\Sigma^{*} ; \mathbb{R}^{3}\right): \operatorname{curl} u=0 \text { in } \Sigma^{*}\right\} .
$$




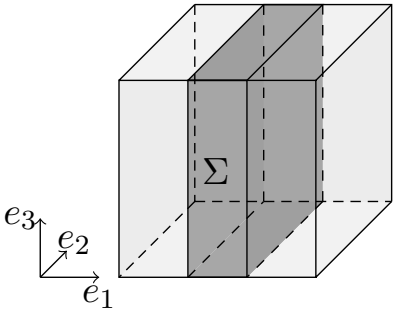

(a)

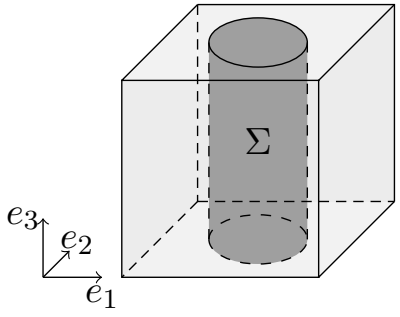

(b)

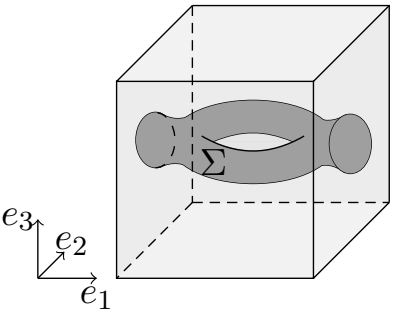

(c)

Figure 1: The above figure shows three admissible microstructures. The cube represents the periodicity cell $Y$ and the dark grey areas illustrate the microstructures $\Sigma$.

The definition will involve the following space of test functions:

$$
V\left(\Sigma^{*}\right):=\left\{\phi \in \mathrm{L}^{2}\left(Y ; \mathbb{R}^{3}\right): \operatorname{curl} \phi=0 \text { in } Y, \phi=0 \text { in } \Sigma\right\} .
$$

Both $X\left(\Sigma^{*}\right)$ and $V\left(\Sigma^{*}\right)$ can be considered as closed subspaces of $\mathrm{L}^{2}\left(\Sigma^{*} ; \mathbb{R}^{3}\right)$ and inherit its Hilbert space structure. We also introduce the space of attainable averages of fields from $V\left(\Sigma^{*}\right)$,

$$
A\left(\Sigma^{*}\right):=\left\{\int_{Y} \phi: \phi \in V\left(\Sigma^{*}\right)\right\} \subset \mathbb{R}^{3} .
$$

We finally have to construct, starting from a linear subspace $U \subset \mathbb{R}^{3}$, a new subspace $U^{\wedge \perp} \subset \mathbb{R}^{3}$ : Given $U \subset \mathbb{R}^{3}$, we introduce the wedge-annihilator $U^{\wedge}:=\left\{b \in \mathbb{R}^{3}: b \wedge x=\right.$ $0 \forall x \in U\}$. The set $U^{\wedge \perp} \subset \mathbb{R}^{3}$ is defined as its orthogonal complement, $U^{\wedge \perp}:=\left(U^{\wedge}\right)^{\perp}$. It turns out that

$$
U^{\wedge \perp}= \begin{cases}\{0\} & \text { if } \operatorname{dim} U=0 \\ U^{\perp} & \text { if } \operatorname{dim} U=1 \\ \mathbb{R}^{3} & \text { if } \operatorname{dim} U \geq 2 .\end{cases}
$$

Definition 2.1 (Geometric average). The geometric average is the unique linear map

$$
\mathcal{G}: X\left(\Sigma^{*}\right) \rightarrow A\left(\Sigma^{*}\right)^{\wedge}, \quad \mathcal{G}(u)=: \oint_{\Sigma^{*}} u,
$$

that satisfies the identity

$$
\int_{\Sigma^{*}} u \wedge \phi=\left(\oint_{\Sigma^{*}} u\right) \wedge\left(\int_{\Sigma^{*}} \phi\right)
$$

for all $u \in X\left(\Sigma^{*}\right)$ and $\phi \in V\left(\Sigma^{*}\right)$. We say that $\oint_{\Sigma^{*}} u$ is the geometric average of $u$.

Remark. The unique vector $\oint_{\Sigma^{*}} u \in A\left(\Sigma^{*}\right)^{\wedge \perp}$ in Definition 2.1 is the one with the minimal Euclidean norm among all vectors $\oint_{\Sigma^{*}} u \in \mathbb{R}^{3}$ satisfying (2.6).

The following theorem establishes the validity of Definition 2.1 by proving existence and uniqueness of $\oint_{\Sigma^{*}} u$. In the proof we use a fact from linear algebra, formulated in Lemma 2.3 after the proof of the theorem.

Theorem 2.2. There exists one and only one map that satisfies (2.5) and (2.6). This map is linear, bounded and onto. 
Proof. Let $u \in X\left(\Sigma^{*}\right)$. For the proof of existence and uniqueness of the map $\mathcal{G}$, we consider the map

$$
B: A\left(\Sigma^{*}\right) \rightarrow \mathbb{R}^{3}, \quad \int_{\Sigma^{*}} \phi \mapsto \int_{\Sigma^{*}} u \wedge \phi \text { for } \phi \in V\left(\Sigma^{*}\right),
$$

and prove that $B$ is well-defined (i.e., independent of the choice of $\phi$ ) and skew-symmetric in the sense that it satisfies $B c \cdot c=0$ for every $c \in A\left(\Sigma^{*}\right)$. Once we have done this, existence and uniquenes of the map $\mathcal{G}$ follows from Lemma 2.3 below.

The map $B$ is well-defined. It suffices to show that $\int_{\Sigma^{*}} u \wedge \phi=0$ holds for all $\phi \in V\left(\Sigma^{*}\right)$ with $\int_{\Sigma^{*}} \phi=0$. Fix such a vector $\phi \in V\left(\Sigma^{*}\right)$. As curl $\phi=0$ in $Y$, we find $f \in \mathrm{H}^{1}(Y)$ and $c \in \mathbb{R}^{3}$ such that $\phi=\operatorname{grad} f+c$ in $Y$. Butthen $\int_{\Sigma^{*}} \phi=0$ implies $c=0$. Moreover, we can choose $f=0$ in $\Sigma$ since $\phi$ vanishes in the connected set $\Sigma$. In particular, $\left.f\right|_{\Sigma^{*}} \in \mathrm{H}_{0}^{1}\left(\Sigma^{*}\right)$ and hence

$$
\int_{\Sigma^{*}} u \wedge \phi=\int_{\Sigma^{*}} u \wedge \operatorname{grad} f=-\int_{\Sigma^{*}} \operatorname{curl}(f u)=0 .
$$

The map $B$ is skew-symmetric. Given $c \in A\left(\Sigma^{*}\right)$, we find $\phi \in V\left(\Sigma^{*}\right)$ such that $\int_{\Sigma^{*}} \phi=c$. For $\phi$ we find $f \in \mathrm{H}^{1}(Y)$ such that $\phi=c+\operatorname{grad} f$ in $Y$ and $\left.f\right|_{\Sigma^{*}} \in H_{0}^{1}\left(\Sigma^{*}\right)$. Observe that curl $u=\operatorname{curl} \phi=0$ in $\Sigma^{*}$ and therefore

$$
\int_{\Sigma^{*}}(u \wedge \phi) \cdot \operatorname{grad} f=\int_{\Sigma^{*}} \operatorname{div}(f(u \wedge \phi))=0 .
$$

Using (2.7), we see that

$$
B c \cdot c=\int_{\Sigma^{*}}(u \wedge \phi) \cdot c=\int_{\Sigma^{*}}(u \wedge \phi) \cdot(c+\operatorname{grad} f)=\int_{\Sigma^{*}}(u \wedge \phi) \cdot \phi=0,
$$

which proves the claim.

It is now a simple fact from linear algebra, written down in Lemma 2.3 below, and proved in the appendix, that these properties of the map $B$ imply the existence and uniqueness of a vector $\oint_{\Sigma^{*}} u \in A\left(\Sigma^{*}\right)^{\wedge \downarrow}$ such that (2.6) holds. As the dependence on $u$ is linear, this defines a linear map $\mathcal{G}: V\left(\Sigma^{*}\right) \rightarrow A\left(\Sigma^{*}\right)^{\wedge \perp}$. It remains to prove that $\mathcal{G}$ is bounded and onto.

The map $\mathcal{G}$ is bounded. Since $A\left(\Sigma^{*}\right)^{\wedge} \downarrow$ is finite dimensional, we only need to show that $\operatorname{ker} \mathcal{G} \subset X\left(\Sigma^{*}\right)$ is closed. Let $\left(u_{k}\right)_{k}$ be a sequence in $\operatorname{ker} \mathcal{G}$ and suppose that $u_{k} \rightarrow u$ in $X\left(\Sigma^{*}\right)$ for some $u \in X\left(\Sigma^{*}\right)$. Then, for every $\phi \in V\left(\Sigma^{*}\right)$ there holds

$$
0=\lim _{k \rightarrow \infty} \int_{\Sigma^{*}} u_{k} \wedge \phi=\int_{\Sigma^{*}} u \wedge \phi .
$$

Thus $\mathcal{G} u=0$ and the claim follows.

The map $\mathcal{G}$ is onto. Given any $c \in A\left(\Sigma^{*}\right)^{\wedge \perp}$, consider the constant function $u \equiv c \in$ $X\left(\Sigma^{*}\right)$. Since $(2.6)$ holds true with $c$ instead of $\oint_{\Sigma^{*}} u$, we conclude that $\oint_{\Sigma^{*}} u=c$.

In the above proof we used the following simple fact from Linear Algebra, a proof of which is provided in Appendix A.

Lemma 2.3 (Linear Algebra fact). Let $U \subset \mathbb{R}^{3}$ be a linear subspace and $B: U \rightarrow \mathbb{R}^{3}$ a linear map such that $B x \cdot x=0$ for every $x \in U$. Then there exists a unique vector $b \in U^{\wedge \downarrow}$ such that

$$
B x=b \wedge x, \quad \text { for all } x \in U .
$$

Remark. The unique vector $b \in U^{\wedge}$ in Lemma 2.3 is the one with the minimal Euclidean norm among all vectors $b \in \mathbb{R}^{3}$ satisfying (2.8). 


\subsection{Properties of the geometric average}

In this subsection, we collect a few basic properties of the geometric average and provide computations for a few simple cases.

Remark. Assume that $A\left(\Sigma^{*}\right)=\operatorname{span}\left\{e_{1}, \ldots, e_{k}\right\}$, where $k \in\{0,1,2,3\}$. Then there exist $\phi_{1}, \ldots, \phi_{k} \in V\left(\Sigma^{*}\right)$ with $\int_{\Sigma^{*}} \phi_{i}=e_{i}$ for $1 \leq i \leq k$. Setting $\phi_{k+1}:=\ldots:=\phi_{3}:=0$, we have the following explicit formula for the geometric average:

$$
\oint_{\Sigma^{*}} u=\left(\begin{array}{c}
\left(\int_{\Sigma^{*}} u \wedge \phi_{2}\right)_{3} \\
-\left(\int_{\Sigma^{*}} u \wedge \phi_{1}\right)_{3} \\
\left(\int_{\Sigma^{*}} u \wedge \phi_{1}\right)_{2}
\end{array}\right) .
$$

Proof. In the case $k=0$ we have $A\left(\Sigma^{*}\right)=\{0\}$ and $A\left(\Sigma^{*}\right)^{\wedge\llcorner}=\{0\}$, hence $\oint_{\Sigma^{*}} u=0$, which is in accordance with (2.9) since $\phi_{1}=\phi_{2}=0$.

In the case $k \geq 1$ we can verify the second and third component of equation (2.9) by explicit computations:

$$
\left(\oint_{\Sigma^{*}} u\right)_{2}=-\left(\left(\oint_{\Sigma^{*}} u\right) \wedge e_{1}\right)_{3}=-\left(\int_{\Sigma^{*}} u \wedge \phi_{1}\right)_{3},
$$

and

$$
\left(\oint_{\Sigma^{*}} u\right)_{3}=\left(\left(\oint_{\Sigma^{*}} u\right) \wedge e_{1}\right)_{2}=\left(\int_{\Sigma^{*}} u \wedge \phi_{1}\right)_{2} .
$$

For the first component, we consider the cases $k=1$ and $k \geq 2$ separately. In the case $k=1$ we have $A\left(\Sigma^{*}\right)=\mathbb{R} e_{1}$ and $A\left(\Sigma^{*}\right)^{\wedge \downarrow}=\{0\} \times \mathbb{R}^{2}$, hence $\left(\oint_{\Sigma^{*}} u\right)_{1}=0$. This is in accordance with (2.9) since $\phi_{2}=0$. In the case $k \geq 2$ we compute

$$
\left(\oint_{\Sigma^{*}} u\right)_{1}=\left(\left(\oint_{\Sigma^{*}} u\right) \wedge e_{2}\right)_{3}=\left(\int_{\Sigma^{*}} u \wedge \phi_{2}\right)_{3} .
$$

Thus (2.9) holds for all $0 \leq k \leq 3$.

Proposition 2.4. Let $\Sigma$ be an admissible microstructure.

(i) In the case $\Sigma=\emptyset$ the geometric average coincides with the volume average. More precisely, for every vector field $u \in \mathrm{L}^{2}\left(Y ; \mathbb{R}^{3}\right)$ with curl $u=0$ in $Y$ there holds

$$
\oint_{Y} u=\int_{Y} u
$$

(ii) For every $f \in \mathrm{H}^{1}\left(\Sigma^{*}\right)$ the vector field $\operatorname{grad} f$ is an element of $X\left(\Sigma^{*}\right)$ with

$$
\oint_{\Sigma^{*}} \operatorname{grad} f=0
$$

(iii) Any constant vector field $c \in \mathbb{R}^{3}$ is an element of $X\left(\Sigma^{*}\right)$ with

$$
\oint_{\Sigma^{*}} c=\pi_{A\left(\Sigma^{*}\right)^{\wedge}}(c)
$$

where $\pi_{A\left(\Sigma^{*}\right)^{\wedge\llcorner}}: \mathbb{R}^{3} \rightarrow \mathbb{R}^{3}$ is the orthogonal projection onto $A\left(\Sigma^{*}\right)^{\wedge}$. 
Proof. (i) Let $u \in \mathrm{L}^{2}\left(Y ; \mathbb{R}^{3}\right)$ be a vector field with curl $u=0$ in $Y$. As every $c \in \mathbb{R}^{3}$ is an element of $V(Y)$, we find that

$$
\left(\oint_{Y} u\right) \wedge c=\int_{Y} u \wedge c=\left(\int_{Y} u\right) \wedge c
$$

As $A\left(\Sigma^{*}\right)^{\wedge \downarrow}=\mathbb{R}^{3}$, the condition $\int_{Y} u \in A\left(\Sigma^{*}\right)^{\wedge \downarrow}$ trivially holds, and the claim is proved.

(ii) Let $f \in \mathrm{H}^{1}\left(\Sigma^{*}\right)$ and let $\phi \in V\left(\Sigma^{*}\right)$ be arbitrary. We choose an $\mathrm{H}^{1}(Y)$-extension $\tilde{f} \in \mathrm{H}^{1}(Y)$ of $f$ and compute

$$
\left(\oint_{\Sigma^{*}} \operatorname{grad} f\right) \wedge\left(\int_{\Sigma^{*}} \phi\right)=\int_{\Sigma^{*}} \operatorname{grad} f \wedge \phi=\int_{Y} \operatorname{grad} \tilde{f} \wedge \phi=\int_{Y} \operatorname{curl}(\tilde{f} \phi)=0 .
$$

The claim follows as $0 \in A\left(\Sigma^{*}\right)^{\wedge \downarrow}$.

(iii) For $c \in \mathbb{R}^{3}$ and $\phi \in V\left(\Sigma^{*}\right)$ we find

$$
\left(\oint_{\Sigma^{*}} c\right) \wedge\left(\int_{\Sigma^{*}} \phi\right)=\int_{\Sigma^{*}} c \wedge \phi=c \wedge\left(\int_{\Sigma^{*}} \phi\right)=\pi_{A\left(\Sigma^{*}\right) \wedge \perp}(c) \wedge\left(\int_{\Sigma^{*}} \phi\right)
$$

where the last equality holds by the definition of $A\left(\Sigma^{*}\right)^{\wedge \downarrow}$ and $\int_{\Sigma^{*}} \phi \in A\left(\Sigma^{*}\right)$.

Next, we investigate how the geometric average is affected when we enlarge the microstructure.

Proposition 2.5. Let $\Sigma_{1}$ and $\Sigma_{2}$ be two admissible microstructures with $\Sigma_{1} \subset \Sigma_{2}$, and let $u \in X\left(\Sigma_{1}^{*}\right)$. Then there holds

$$
\left.\oint_{\Sigma_{2}^{*}} u\right|_{\Sigma_{2}^{*}}=\pi_{A\left(\Sigma_{2}^{*}\right)} \wedge \downarrow\left(\oint_{\Sigma_{1}^{*}} u\right),
$$

where $\pi_{A\left(\Sigma_{2}^{*}\right)} \wedge\left\llcorner: \mathbb{R}^{3} \rightarrow \mathbb{R}^{3}\right.$ is the orthogonal projection onto $A\left(\Sigma_{2}^{*}\right)^{\wedge \perp}$.

In particular, if $A\left(\Sigma_{1}^{*}\right)^{\wedge \perp}=A\left(\Sigma_{2}^{*}\right)^{\wedge \downarrow}$ we have

$$
\left.\oint_{\Sigma_{2}^{*}} u\right|_{\Sigma_{2}^{*}}=\oint_{\Sigma_{1}^{*}} u
$$

Proof. Let $c \in A\left(\Sigma_{2}^{*}\right)$. Then there exist $\phi \in V\left(\Sigma_{2}^{*}\right)$ and a function $f \in \mathrm{H}^{1}(Y)$ such that $\phi=c+\operatorname{grad} f$ in $Y$. As $\phi=0$ in $\Sigma_{2}$ and $\Sigma_{1} \subset \Sigma_{2}$ we infer that $\phi \in V\left(\Sigma_{1}^{*}\right)$.

$$
\begin{aligned}
\left(\pi_{A\left(\Sigma_{2}^{*}\right) \wedge}\left(\oint_{\Sigma_{1}^{*}} u\right)\right) \wedge c & =\left(\oint_{\Sigma_{1}^{*}} u\right) \wedge c=\int_{\Sigma_{1}^{*}} u \wedge \phi \\
& =\left.\int_{\Sigma_{2}^{*}} u\right|_{\Sigma_{2}^{*}} \wedge \phi=\left(\left.\oint_{\Sigma_{2}^{*}} u\right|_{\Sigma_{2}^{*}}\right) \wedge c .
\end{aligned}
$$

As $c \in A\left(\Sigma_{2}^{*}\right)$ was arbitrary, the claim follows.

Lemma 2.6. Let $\Sigma$ be an admissible microstructure that is compactly contained in $(0,1)^{3}$. Then $A\left(\Sigma^{*}\right)=\mathbb{R}^{3}, A\left(\Sigma^{*}\right)^{\wedge}=\mathbb{R}^{3}$, and $\oint_{\Sigma^{*}} u$ is the unique vector in $\mathbb{R}^{3}$ that satisfies $(1.1)$ for all $\phi \in V\left(\Sigma^{*}\right)$. 
Proof. For $c \in \mathbb{R}^{3}$ we consider the function $g: \Sigma \rightarrow \mathbb{R}, g(y):=-y \cdot c$, which is an element of $\mathrm{H}^{1}(\Sigma)$, since $\Sigma$ does not touch the boundaries of the cube. Let $f \in \mathrm{H}^{1}(Y)$ be the solution to the Dirichlet problem

$$
\begin{aligned}
& -\Delta f=0 \quad \text { in } \Sigma^{*}, \\
& f=g \quad \text { in } \Sigma \text {. }
\end{aligned}
$$

We set $\phi:=\operatorname{grad} f+c$. The function $\phi$ is an element of $V\left(\Sigma^{*}\right)$ with the average $c$.

Remark (Examples from Figure 1). Let us discuss the spaces $V\left(\Sigma^{*}\right), A\left(\Sigma^{*}\right)$, and $A\left(\Sigma^{*}\right)^{\wedge \downarrow}$ for the three microstructures of Figure 1.

(a) Let $\gamma \in(0,1 / 2)$ and define

$$
\Sigma:=\left\{\left(y_{1}, y_{2}, y_{3}\right) \in Y: y_{1} \in\left(\frac{1}{2}-\gamma, \frac{1}{2}+\gamma\right)\right\} .
$$

The space $V\left(\Sigma^{*}\right)$ contains the function $\phi_{1}:=\left|\Sigma^{*}\right|^{-1} e_{1} \mathbb{1}_{\Sigma^{*}}$. The tangential trace of a vector field in $V\left(\Sigma^{*}\right)$ does not jump across $\partial \Sigma$, which can be used to show $A\left(\Sigma^{*}\right)=\mathbb{R} e_{1}$, compare the definition in (2.3). This implies $A\left(\Sigma^{*}\right)^{\wedge \perp}=\operatorname{span}\left\{e_{2}, e_{3}\right\}$. We further conclude by (2.9) that for every $u \in X\left(\Sigma^{*}\right)$ there holds:

$$
\oint_{\Sigma^{*}} u=\left(\begin{array}{c}
0 \\
-\left(\int_{\Sigma^{*}} u \wedge \phi_{1}\right)_{3} \\
\left(\int_{\Sigma^{*}} u \wedge \phi_{1}\right)_{2}
\end{array}\right)=\left(\begin{array}{c}
0 \\
f_{\Sigma^{*}} u_{2} \\
f_{\Sigma^{*}} u_{3}
\end{array}\right) .
$$

(b) For a fixed radius $r \in(0,1 / 2)$ we set

$$
\Sigma:=\left\{\left(y_{1}, y_{2}, y_{3}\right) \in Y:\left(y_{1}-\frac{1}{2}\right)^{2}+\left(y_{2}-\frac{1}{2}\right)^{2}<r^{2}\right\} .
$$

We have no explicit formula for basis functions of $V\left(\Sigma^{*}\right)$. Nevertheless, one can easily see that averages in $e_{2}$ and $e_{3}$ direction can be realized by solving two-dimensional elliptic equations. We find that $A\left(\Sigma^{*}\right)=\mathbb{R} e_{1}+\mathbb{R} e_{2}$. Consequently, there holds $A\left(\Sigma^{*}\right)^{\wedge}=\mathbb{R}^{3}$ by $(2.4)$.

(c) In the geometry with a torus that touches opposite faces, we have that $A\left(\Sigma^{*}\right)=$ $\mathbb{R} e_{2}+\mathbb{R} e_{3}$ and thus $A\left(\Sigma^{*}\right)^{\wedge \perp}=\mathbb{R}^{3}$.

\subsection{Comparison to other definitions}

In this section we compare our definition of the geometric average with the ones already present in the literature.

The first definition appeared in the work of Bouchitté, Bourel, and Felbacq [3]. It was also used, e.g., in [16]. As the notion of a geometric average turned out to be useful in the homogenization of Maxwell's equations, it was later generalised to more general geometries in [18] and [22].

\subsubsection{Microstructures that are compactly contained in the unit cube}

In $[3,4,18]$ the authors consider a microstructure $\Sigma \subset Y$ which is not only connected but also compactly contained in $(0,1)^{3}$. The notion of a geometric average which they introduce satisfies our defining formula (2.6). By Lemma 2.6 we know that the assumption 
on $\Sigma$ implies $A\left(\Sigma^{*}\right)=\mathbb{R}^{3}$. Hence, formula (2.6) uniquely defines the geometric average. It follows that the notion of a geometric average introduced in $[3,4,18]$ is contained in our definition of the geometric average.

To be more precise, in [3] it is further assumed that $\Sigma^{*}$, considered as a subset of the unit cell $(0,1)^{3}$, is simply connected. The authors then define the geometric average by the identity

$$
\int_{\Sigma^{*}} u \cdot \psi=\left(\oint_{\Sigma^{*}} u\right) \cdot\left(\int_{\Sigma^{*}} \psi\right)
$$

which should hold for all $\psi \in \mathrm{L}^{2}\left(Y ; \mathbb{R}^{3}\right)$ with $\operatorname{div} \psi=0$ in $Y$ and $\psi=0$ in $\Sigma$. Given any $\phi \in V\left(\Sigma^{*}\right)$ and $c \in \mathbb{R}^{3}$, one easily verifies that $\psi:=\phi \wedge c$ has these properties (in particular, curl $\phi=0$ implies div $\psi=0$ in $Y$ ). Inserting $\psi$ into (2.10) for $c \in\left\{e_{1}, e_{2}, e_{3}\right\}$, one recovers condition (2.6).

In [3] it is also remarked (still assuming that $\Sigma^{*}$ is simply connected in $\left.(0,1)^{3}\right)$ that any $u \in X\left(\Sigma^{*}\right)$ can be written in the form $u=c+\operatorname{grad} g$ for some $c \in \mathbb{R}^{3}$ and $g \in \mathrm{H}^{1}\left(\Sigma^{*}\right)$, and it holds $c=\oint_{\Sigma^{*}} u$.

In [4] it is remarked that one can interprete $\oint_{\Sigma^{*}} u$ as given by line integrals: Suppose $u \in X\left(\Sigma^{*}\right)$ is continuous, and $\gamma:[0,1] \rightarrow \Sigma^{*}$ is a continuous path joining opposite faces of $\Sigma^{*}$, that is, $\gamma(1)-\gamma(0)=e_{k}$ for some $k \in\{1,2,3\}$, then

$$
\left(\oint_{\Sigma^{*}} u\right) \cdot e_{k}=\int_{\gamma} u \cdot \mathrm{d} s .
$$

The value of this integral is independent of the choice of $\gamma$ since $u$ is curl-free and $\Sigma^{*}$ is simply connected.

In [18] the authors drop the assumption that $\Sigma^{*}$ has to be simply connected. They define the geometric average by (2.11), that is, in terms of line integrals. In order to cope with the possible non-uniqueness arising from the missing simple connectedness of $\Sigma^{*}$, they make the specific choice $\gamma \in\left\{\gamma_{1}, \gamma_{2}, \gamma_{3}\right\}$, where $\gamma_{k}(t):=t e_{k}$ for $t \in[0,1]$.

\subsubsection{Microstructures touching the boundary of the unit cube}

Two authors of this article suggested a definition of the geometric average that applies for microstructures $\Sigma$ which are connected, but not necessarily compactly contained in $(0,1)^{3}$; see [22]. They assume that $\Sigma$ belongs to the class of microstructures defined by the following property: every $u \in X\left(\Sigma^{*}\right)$ admits a decomposition $u=c+\operatorname{grad} g$ in $\Sigma^{*}$ for some $c \in \mathbb{R}^{3}$ and $g \in \mathrm{H}^{1}\left(\Sigma^{*}\right)$.

In the setting of [4], the vector $c \in \mathbb{R}^{3}$ was uniquely determined. However, when $\Sigma$ connects opposite faces of the cube $(0,1)^{3}$, this uniqueness is no longer granted. Using the notion of $k$-loops, the authors of [22] select the components of $c$ that are uniquely determined. We would like to sketch the construction here, which gives us the opportunity to correct the definition of the geometric average that was given in [22] (there exist microstructures $\Sigma$ for which the original definition cannot be used).

Given a vector $k \in \mathbb{Z}^{3}$, a closed loop $\gamma:[0,1] \rightarrow \Sigma^{*}$ is called a $k$-loop if one (and then every) lift $\tilde{\gamma}:[0,1] \rightarrow \mathbb{R}^{3}$ of $\gamma$ with respect to the canonical projection $\mathbb{R} \rightarrow \mathbb{R} / \mathbb{Z} \cong Y$ satisfies $\tilde{\gamma}(1)-\tilde{\gamma}(0)=k$. Define the space

$$
\mathcal{L}_{\Sigma^{*}}:=\operatorname{span}_{\mathbb{R}}\left\{k \in \mathbb{Z}^{3}: \text { there is a } k \text {-loop } \gamma:[0,1] \rightarrow \Sigma^{*}\right\},
$$

and denote by $\pi_{\Sigma^{*}}: \mathbb{R}^{3} \rightarrow \mathbb{R}^{3}$ the orthogonal projection onto $\mathcal{L}_{\Sigma^{*}}$. The geometric average of $u=c+\operatorname{grad} g$ is then defined as $\oint_{\Sigma^{*}} u:=\pi_{\Sigma^{*}}(c)$. 
In [22] the geometric average is correctly defined if $\mathcal{L}_{\Sigma^{*}}$ is spanned by vectors of the form $n_{k} e_{k}\left(k \in\{1,2,3\}, n_{k} \in \mathbb{N}\right)$ for which there exist $\left(n_{k} e_{k}\right)$-loops in $\Sigma^{*}$. This is true for all the examples discussed in Section 5 of [22] but not for general microstructures $\Sigma$.

As the notion introduced in [22] satisfies (2.10), we can conclude (as above) that in the case $\operatorname{dim} A\left(\Sigma^{*}\right) \geq 2$ this notion is contained in our definition of the geometric average.

In the case $\operatorname{dim} A\left(\Sigma^{*}\right)=0$ the two notions do not necessarily coincide. We provide the following example: Choosing

$$
\Sigma^{*}:=\left\{\left(y_{1}, y_{2}, y_{3}\right) \in Y:\left(y_{1}-1 / 2\right)^{2}+\left(y_{2}-1 / 2\right)^{2}<1 / 4\right\},
$$

we find that the constant vector field $u \equiv e_{3}$ is an element of $X\left(\Sigma^{*}\right)$. As there is an $e_{3}$-loop in $\Sigma^{*}$, we conclude that the geometric agerage of $u$ in the sense of [22] is $e_{3}$. However, since $A\left(\Sigma^{*}\right)=\{0\}$, we know that the geometric average of $u$ as defined in the present paper vanishes.

\section{Geometric average and two-scale convergence}

In this section, we investigate the geometric average in the context of periodic homogenization. A classical task is to consider sequences of functions $u^{\eta}$ in a periodic geometry and to derive effective equations for limits of these functions. The fundamental tool for this procedure is two-scale convergence. Here, we are interested in sequences $u^{\eta}$ of vector fields that vanish inside the microstructure and that have a bounded curl. Such sequences naturally appear in the study of the time harmonic Maxwell equations (see Section 5).

Our main interest is the homogenization in a situation that two different microstructures are used in two different parts of the domain. At the interface of the two subdomains, we expect that certain components of the limiting field satisfy continuity properties. In this context, the geometric average plays an important role, since trace properties can only be exected for the limit field that is defined via the geometric average.

Let $\Omega$ denote any open subset of $\mathbb{R}^{3}, \Omega_{1} \subset \Omega$ an open subset and $\Omega_{2}=\Omega \backslash \bar{\Omega}_{1}$ the complementary open subdomain.

In the two subdomains, we want to use two different microstructures. Let therefore two admissible microstructures be given by $\Sigma_{1}, \Sigma_{2} \subset Y$. As before, the complementary open sets are denoted by $\Sigma_{i}^{*}:=Y \backslash \bar{\Sigma}_{i}$. The case $\Sigma_{i}=\emptyset$ is permitted and accounts for the absence of microstructure in $\Omega_{i}$. For $\eta>0$ we define the index sets

$$
\mathcal{K}:=\left\{k \in \mathbb{Z}^{3}: \eta k \in \Omega\right\}, \quad \text { and } \quad \mathcal{K}_{i}:=\left\{k \in \mathbb{Z}^{3}: \eta k \in \Omega_{i}\right\}
$$

These allow us to define the perforated domain by

$$
\Omega_{\eta}:=\Omega \cap\left(\bigcup_{k \in \mathcal{K}_{1}} \eta\left(k+\Sigma_{1}\right) \cup \bigcup_{k \in \mathcal{K}_{2}} \eta\left(k+\Sigma_{2}\right)\right) \quad \text { and } \quad \Omega_{\eta}^{*}:=\Omega \backslash \bar{\Omega}_{\eta} .
$$

We will see that the study of interfaces requires also a study of the interaction of the two microstructure. The relevant quantity turns out to be the union of the two microstructures. We set

$$
\Sigma:=\Sigma_{1} \cup \Sigma_{2} \quad \text { and } \quad \Sigma^{*}:=Y \backslash \bar{\Sigma} \text {. }
$$


We keep in mind that we have the inclusions

$$
\Sigma_{i} \subset \Sigma, \quad \Sigma^{*} \subset \Sigma_{i}^{*}, \quad V\left(\Sigma^{*}\right) \subset V\left(\Sigma_{i}^{*}\right),
$$

and the restriction map

$$
X\left(\Sigma_{i}^{*}\right) \rightarrow X\left(\Sigma^{*}\right),\left.\quad u \mapsto u\right|_{\Sigma^{*}}
$$

\subsection{The geometric average in effective equations}

In the subsequent theorem we use two-scale convergence, which we denote with $\stackrel{2}{\longrightarrow}$.

Theorem 3.1. Let $\left(u^{\eta}\right)_{\eta}$ be a sequence in $\mathrm{L}^{2}\left(\Omega ; \mathbb{R}^{3}\right)$ with the two properties

$$
\begin{aligned}
u^{\eta} & =0 \text { in } \Omega_{\eta}, \\
w^{\eta} & :=\operatorname{curl}\left(\left.u^{\eta}\right|_{\Omega_{\eta}^{*}}\right) \in \mathrm{L}^{2}\left(\Omega_{\eta}^{*} ; \mathbb{R}^{3}\right) .
\end{aligned}
$$

We extend $w^{\eta}$ by 0 to all of $\Omega$ and assume that $u^{\eta} \stackrel{2}{\longrightarrow} u_{0}$ and $w^{\eta} \stackrel{2}{\longrightarrow} w_{0}$ for some limit functions $u_{0}, w_{0} \in \mathrm{L}^{2}\left(\Omega \times Y ; \mathbb{R}^{3}\right)$. Then $u_{0}(x, \cdot) \in X\left(\Sigma_{i}^{*}\right)$ for almost every $x \in \Omega_{i}$. Furthermore, if we define the geometric limit by $\hat{u}(x):=\oint_{\Sigma_{i}^{*}} u_{0}(x, \cdot)$ for $x \in \Omega_{i}$, there holds

$$
\operatorname{curl} \hat{u} \cdot \int_{\Sigma_{i}^{*}} \phi=\int_{\Sigma_{i}^{*}} w_{0} \cdot \phi \quad \text { for all } \phi \in V\left(\Sigma_{i}^{*}\right),
$$

in the sense of distributions on $\Omega_{i}$. Globally, i.e. on $\Omega$, there holds:

$$
\operatorname{curl} \hat{u} \cdot \int_{\Sigma^{*}} \phi=\int_{\Sigma^{*}} w_{0} \cdot \phi \quad \text { for all } \phi \in V\left(\Sigma^{*}\right),
$$

in the sense of distributions on $\Omega$.

Proof. The $\mathrm{L}^{2}$-bound on the sequence $\left(\left.\operatorname{curl} u^{\eta}\right|_{\Omega_{\eta}^{*}}\right)_{\eta}$ implies, by standard arguments in the theory of two-scale convergence, that $\operatorname{curl} u_{0}(x, \cdot)=0$ holds in $\Sigma_{i}^{*}$ for almost all $x \in \Omega_{i}$. This implies $u_{0}(x, \cdot) \in X\left(\Sigma_{i}^{*}\right)$ for almost all $x \in \Omega_{i}$.

In order to show (3.2), we choose a macroscopic test function $\theta \in C_{c}^{\infty}\left(\Omega_{i}\right)$ and a microscopic test function $\phi \in V\left(\Sigma_{i}^{*}\right)$. For $\eta>0$ we set $\psi(x, y):=\theta(x) \phi(y)$ and $\psi^{\eta}(x):=\psi(x, x / \eta)$. As $\left(w^{\eta}\right)_{\eta}$ two-scale converges to $w_{0}$, we find, by the definition of two-scale convergence,

$$
\lim _{\eta \rightarrow 0} \int_{\Omega} w^{\eta}(x) \cdot \psi^{\eta}(x) \mathrm{d} x=\int_{\Omega} \theta(x)\left(\int_{Y} w_{0}(x, y) \cdot \phi(y) \mathrm{d} y\right) \mathrm{d} x .
$$

On the other hand, as curl $\phi=0$ in $Y$, there holds curl $\psi^{\eta}(x)=\operatorname{grad} \theta(x) \wedge \phi(x / \eta)$ for all $x \in \Omega_{i}$. Using this and (3.1), we can compute the term also differently:

$$
\begin{aligned}
\lim _{\eta \rightarrow 0} \int_{\Omega} w^{\eta}(x) \cdot \psi^{\eta}(x) \mathrm{d} x & =\lim _{\eta \rightarrow 0} \int_{\Omega_{\eta}^{*}} \operatorname{curl} u^{\eta}(x) \cdot \psi^{\eta}(x) \mathrm{d} x \\
& =\lim _{\eta \rightarrow 0} \int_{\Omega_{\eta}^{*}} u^{\eta}(x) \cdot(\operatorname{grad} \theta(x) \wedge \phi(x / \eta)) \mathrm{d} x \\
& =-\int_{\Omega} \operatorname{grad} \theta(x) \cdot\left(\int_{\Sigma_{i}^{*}} u_{0}(x, y) \wedge \phi(y) \mathrm{d} y\right) \mathrm{d} x \\
& =-\int_{\Omega} \operatorname{grad} \theta(x) \cdot\left(\hat{u}(x) \wedge \int_{\Sigma_{i}^{*}} \phi(y) \mathrm{d} y\right) \mathrm{d} x .
\end{aligned}
$$


Comparing (3.4) and (3.5), recalling that $\theta$ was arbitrary and that $\int_{Y} \phi(y) \mathrm{d} y$ is a constant, we obtain equation (3.2).

In order to derive equation (3.3), we argue with a new interpretation of equation (3.2). We consider the union $\Sigma:=\Sigma_{1} \cup \Sigma_{2}$ of the two microstructures and a new domain $\Omega_{\eta}^{\Sigma}$, which is obtained from the microstructure $\Sigma$. Then $\left(u^{\eta}\right)_{\eta}$ and $\left(w^{\eta}\right)_{\eta}$ satisfy all prerequisites of the theorem with the single perforation type $\Sigma$ (the functions are restricted to a smaller set). Proposition 2.5 implies that the geometric average of the twoscale limit $\hat{u}^{\Sigma}$ satisfies

$$
\widehat{u}^{\Sigma}=\pi_{A\left(\Sigma^{*}\right)^{\wedge \perp}}(\hat{u}) .
$$

Relation (3.2) can be used with $\Sigma$ on all of $\Omega$ and provides

$$
\operatorname{curl}\left(\pi_{A\left(\Sigma^{*}\right)^{\wedge \perp}}(\hat{u})\right) \cdot \int_{\Sigma^{*}} \phi=\operatorname{curl} \hat{u}^{\Sigma} \cdot \int_{\Sigma^{*}} \phi=\int_{\Sigma^{*}} w_{0} \cdot \phi
$$

for all $\phi \in V\left(\Sigma^{*}\right)$.

We claim that any two vectors $a, c \in \mathbb{R}^{3}$ with $a \in A\left(\Sigma^{*}\right)$ satisfy

$$
\pi_{A\left(\Sigma^{*}\right)^{\wedge \perp}}(c) \wedge a=c \wedge a .
$$

Indeed, the difference of the two sides is $\left(c-\pi_{A\left(\Sigma^{*}\right)} \wedge(c)\right) \wedge a$ where the term in brackets is an element of $\left(A\left(\Sigma^{*}\right)^{\wedge \perp}\right)^{\perp}=\left(\left(A\left(\Sigma^{*}\right)^{\wedge}\right)^{\perp}\right)^{\perp}=A\left(\Sigma^{*}\right)^{\wedge}$. This implies that the wedge product with $a \in A\left(\Sigma^{*}\right)$ vanishes, and (3.7) is shown.

With the help of (3.7) we can calculate for the left-hand side of (3.6) with $a:=\int_{\Sigma^{*}} \phi$

$$
\operatorname{curl}\left(\pi_{A\left(\Sigma^{*}\right)^{\wedge \perp}}(\hat{u})\right) \cdot a=\nabla \cdot\left(\left(\pi_{A\left(\Sigma^{*}\right)^{\wedge \perp}}(\hat{u})\right) \wedge a\right)=\nabla \cdot((\hat{u}) \wedge a)=\operatorname{curl}(\hat{u}) \cdot a .
$$

This proves (3.3).

Corollary 3.2. In the situation of Theorem 3.1,

$$
\pi_{A\left(\Sigma^{*}\right)}(\operatorname{curl} \hat{u}) \in \mathrm{L}^{2}\left(\Omega ; \mathbb{R}^{3}\right),
$$

where $\pi_{A\left(\Sigma^{*}\right)}: \mathbb{R}^{3} \rightarrow \mathbb{R}^{3}$ is the orthogonal projection onto $A\left(\Sigma^{*}\right)$. In particular, if $\Gamma:=\bar{\Omega}_{1} \cap \bar{\Omega}_{2}$ is a smooth two-dimensional surface in $\Omega$, certain tangential components of $\hat{u}$ possess a trace on $\Gamma$; the subspace $A\left(\Sigma^{*}\right)$ determines, which components.

Proof. For any $c \in A\left(\Sigma^{*}\right)$ and a corresponding function $\phi \in V\left(\Sigma^{*}\right)$ with $c=\int_{Y} \phi$, the right hand side of (3.3) is an element of $\mathrm{L}^{2}(\Omega)$, hence also curl $\hat{u} \cdot c \in \mathrm{L}^{2}(\Omega)$.

\subsection{Convergence of tangential traces}

In this subsection, we consider the special case $\Omega_{1}=\Omega \cap\left\{x_{1}>0\right\}$. When we assume $e_{2} \in A\left(\Sigma^{*}\right)$, then Corollary 3.2 shows that the component $\hat{u}_{3}$ possesses a trace on $\Gamma:=\Omega \cap\left\{x_{1}=0\right\}$. In particular, $\hat{u}_{3}$ does not jump across $\Gamma$. Indeed, for any test function $\theta \in C_{c}^{\infty}(\Omega)$, an integration by parts shows that

$$
\int_{\Omega_{1}} \operatorname{curl}(\theta \hat{u}) \cdot e_{2}=\int_{\Gamma}\left(-e_{1} \wedge \theta \hat{u}\right) \cdot e_{2}=\int_{\Gamma} \theta \hat{u}_{3} .
$$

Even more can be said in the above situation: We can analyze the limit of traces of $u_{3}^{\eta}$. This is the result of the next proposition. 
Proposition 3.3 (Convergence of tangential traces). We consider the situation of Theorem 3.1: $\Omega_{\eta}$ is constructed from $\Omega$ with $\Sigma_{i}$ and $\Sigma$, the sequences $\left(u^{\eta}\right)_{\eta}$ and $\left(w^{\eta}\right)_{\eta}$ satisfy all the properties of Theorem 3.1. We assume $\Omega_{1}=\Omega \cap\left\{x_{1}>0\right\}$ and $e_{2} \in A\left(\Sigma^{*}\right)$, and demand that $\left\{y_{1}=0\right\} \subset \Sigma^{*}$. We use $\hat{u}(x):=\oint_{\Sigma^{*}} u_{0}(x, \cdot)$ for all $x \in \Omega$.

Then

$$
\left.\left.u_{3}^{\eta}\right|_{\Gamma} \rightarrow \hat{u}_{3}\right|_{\Gamma}
$$

in the sense of distributions on $\Gamma:=\Omega \cap\left\{x_{1}=0\right\}$, where $\left.u_{3}^{\eta}\right|_{\Gamma}$ and $\left.\hat{u}_{3}\right|_{\Gamma}$ are to be understood in the sense of traces.

Proof. Consider any test function $\theta \in C_{c}^{\infty}(\Omega)$. Since $e_{2} \in A\left(\Sigma^{*}\right)$, there exists a function $f \in \mathrm{H}^{1}(Y)$ such that $e_{2}+\operatorname{grad} f \in V\left(\Sigma^{*}\right)$. Let $g \in \mathrm{H}^{1}(Y)$ be any function that vanishes in a neighborhood of $\Sigma$, satisfying $f+g=0$ on $\left\{y_{1}=0\right\}$. Then $\phi:=e_{2}+\operatorname{grad}(f+g) \in V\left(\Sigma^{*}\right)$. The tangential trace of $\phi$ on $\left\{y_{1}=0\right\}$ equals $e_{2}$, it vanishes on $\partial \Sigma^{*}$. We write $\phi^{\eta}:=\phi(\cdot / \eta)$, use integration by parts and exploit the two-scale convergence of $\left(u^{\eta}\right)_{\eta}$ and $\left(w^{\eta}\right)_{\eta}$ to find

$$
\begin{aligned}
\int_{\Gamma} \theta u_{3}^{\eta} & =\int_{\Gamma}\left(-e_{1} \wedge \theta u^{\eta}\right) \cdot e_{2}^{\eta}=\int_{\Gamma}\left(\nu \wedge \theta u^{\eta}\right) \cdot \phi^{\eta} \\
& =\int_{\Omega_{+} \cap \Omega_{\eta}^{*}} \operatorname{curl}\left(\theta u^{\eta}\right) \cdot \phi^{\eta}=\int_{\Omega_{+}} \nabla \theta \cdot\left(u^{\eta} \wedge \phi^{\eta}\right)+\theta w^{\eta} \cdot \phi^{\eta} \\
& \rightarrow \int_{\Omega_{+}} \nabla \theta \cdot \int_{Y} u_{0} \wedge \phi+\int_{\Omega_{+}} \theta \int_{Y} w_{0} \cdot \phi \\
& =\int_{\Omega_{+}} \nabla \theta \cdot \hat{u} \wedge e_{2}+\int_{\Omega_{+}} \theta \int_{Y} w_{0} \cdot \phi .
\end{aligned}
$$

We use (3.3) of Theorem 3.1 and conclude

$$
\begin{aligned}
\lim _{\eta \rightarrow 0} \int_{\left\{x_{1}=0\right\}} \theta u_{3}^{\eta} & =\int_{\Omega_{+}}(\nabla \theta \wedge \hat{u}) \cdot e_{2}+\theta \operatorname{curl} \hat{u} \cdot e_{2} \\
& =\int_{\Omega_{+}} \operatorname{curl}(\theta \hat{u}) \cdot e_{2}=\int_{\left\{x_{1}=0\right\}} \theta u_{3}^{\eta} .
\end{aligned}
$$

This was the claim in (3.8).

\section{De Rham theory and cell problems}

In this section, we revisit the definition of the geometric average and state it in terms of de Rham cohomology groups. We will see that the geometric average is a function of cohomology classes, and that the test functions can also be grouped in cohomology classes. It will turn out that the solutions to the well-known cell problems for the $H$-field and the $E$-field constitute sets of representatives for these cohomology classes.

\subsection{Cohomology groups and a special wedge product}

We have to introduce a few elements of de Rham theory. Given any open subset $U \subset Y$, the first de Rham cohomology group of $U$ is the quotient space

$$
\mathrm{H}_{\mathrm{dR}}^{1}(U):=\left\{u \in \mathrm{L}^{2}\left(U ; \mathbb{R}^{3}\right): \operatorname{curl} u=0\right\} / \sim
$$


where $u \sim u^{\prime}$ whenever there is a function $f \in \mathrm{H}^{1}(U)$ such that $u-u^{\prime}=\operatorname{grad} f$. The second de Rham cohomology group of $U$ is the quotient space

$$
\mathrm{H}_{\mathrm{dR}}^{2}(U):=\left\{v \in \mathrm{L}^{2}\left(U ; \mathbb{R}^{3}\right): \operatorname{div} v=0\right\} / \sim,
$$

where $v \sim v^{\prime}$ whenever there is a vector field $w \in \mathrm{L}^{2}\left(U ; \mathbb{R}^{3}\right)$ such that $v-v^{\prime}=\operatorname{curl} w$.

Given two open subsets $V \subset U \subset Y$, the first de Rham cohomology group of $U$ relative to $V$ is the quotient space

$$
\mathrm{H}_{\mathrm{dR}}^{1}(U, V):=\left\{u \in \mathrm{L}^{2}\left(U ; \mathbb{R}^{3}\right): \operatorname{curl} u=0 \text { in } U, u=0 \text { in } V\right\} / \sim,
$$

where $u \sim u^{\prime}$ whenever there is a function $f \in \mathrm{H}^{1}(U)$ such that $u-u^{\prime}=\operatorname{grad} f$ in $U$ and $f=0$ in $V$. The condition $f=0$ in $V$ is dispensable if $V$ is connected. As $\Sigma$ is connected, this implies in the case $(U, V)=(Y, \Sigma)$ that there is a well-defined inclusion map

$$
i: \mathrm{H}_{\mathrm{dR}}^{1}(Y, \Sigma) \rightarrow \mathrm{H}_{\mathrm{dR}}^{1}(Y), \quad[\phi] \rightarrow[\phi] .
$$

We also remark that there are the following well-known isomorphisms (which we take as identities):

$$
\mathrm{H}_{\mathrm{dR}}^{k}(Y) \rightarrow \mathbb{R}^{3}, \quad[u] \mapsto \int_{Y} u, \quad k \in\{1,2\} .
$$

Being acquainted with some of the language of de Rham theory, we can now introduce the following wedge product which lies at the heart of our definition of the geometric average.

Lemma 4.1. There is a well-defined product

$$
a: \mathrm{H}_{\mathrm{dR}}^{1}\left(\Sigma^{*}\right) \times \mathrm{H}_{\mathrm{dR}}^{1}(Y, \Sigma) \rightarrow \mathrm{H}_{\mathrm{dR}}^{2}(Y), \quad a([u],[\phi]):=[u \wedge \phi],
$$

where, on the right-hand side, $u$ is arbitrarily extended to all of $Y$.

Proof. Let $[u] \in \mathrm{H}_{\mathrm{dR}}^{1}\left(\Sigma^{*}\right)$ and $[\phi] \in \mathrm{H}_{\mathrm{dR}}^{1}(Y, \Sigma)$ be arbitrary. We will extend $u$ to all of $Y$; as $\phi=0$ in $\Sigma$, the choice of the extension does not effect the product $u \wedge \phi$. Our aim is to prove that $\operatorname{div}(u \wedge \phi)=0$ in $Y$. In order to show this fact, we extend $u$ to a function $u \in \mathrm{L}^{2}\left(Y ; \mathbb{R}^{3}\right)$ such that curl $u \in \mathrm{L}^{2}\left(Y ; \mathbb{R}^{3}\right)$. Using that curl $u=0$ in $\Sigma^{*}, \phi=0$ in $\Sigma$, and curl $\phi=0$ in $Y$, we then find that

$$
\operatorname{div}(u \wedge \phi)=(\operatorname{curl} u) \cdot \phi-u \cdot(\operatorname{curl} \phi)=0,
$$

proving that $u \wedge \phi$ represents a de Rham class $[u \wedge \phi] \in \mathrm{H}_{\mathrm{dR}}^{2}(Y)$.

To conclude the proof that the product is well-defined, we have to show that $[u \wedge \phi]=0$ whenever $[u]=0$ or $[\phi]=0$.

In the first case, $u=\operatorname{grad} f$ for some $f \in \mathrm{H}^{1}\left(\Sigma^{*}\right)$. We extend $f$ to a function $f \in \mathrm{H}^{1}(Y)$ and compute

$$
[\operatorname{grad} f \wedge \phi]=\int_{Y} \operatorname{grad} f \wedge \phi=\int_{Y} \operatorname{curl}(f \phi)=0 .
$$

In the second case, $\phi=\operatorname{grad} g$ for some $g \in \mathrm{H}^{1}(Y)$ with $g=0$ in $\Sigma^{*}$. Then

$$
[u \wedge \operatorname{grad} g]=\int_{Y} u \wedge \operatorname{grad} g=-\int_{Y} \operatorname{curl}(g u)=0,
$$

where we used that $g=0$ in $\Sigma$ to obtain the second equality. 
We next restate the definition of the geometric average in a form that makes use of the language that we acquired in this section.

Definition 4.2 (Geometric average - cohomological version). Consider the spaces

$$
A\left(\Sigma^{*}\right):=i \mathrm{H}_{\mathrm{dR}}^{1}(Y, \Sigma) \subset \mathrm{H}_{\mathrm{dR}}^{1}(Y), \quad A\left(\Sigma^{*}\right)^{\wedge \perp} \subset \mathrm{H}_{\mathrm{dR}}^{1}(Y) .
$$

The geometric average is the linear map

$$
\oint_{\Sigma^{*}}: \mathrm{H}_{\mathrm{dR}}^{1}\left(\Sigma^{*}\right) \rightarrow A\left(\Sigma^{*}\right)^{\wedge \downarrow}, \quad[u] \mapsto \oint_{\Sigma^{*}}[u]
$$

that satisfies

$$
[u \wedge \phi]=\left(\oint_{\Sigma^{*}}[u]\right) \wedge i[\phi]
$$

for all $[u] \in \mathrm{H}_{\mathrm{dR}}^{1}\left(\Sigma^{*}\right)$ and $[\phi] \in \mathrm{H}_{\mathrm{dR}}^{1}(Y, \Sigma)$.

\subsection{Cell problems}

We now introduce the $E$-cell problem and the $H$-cell problem. These appear in the homogenization of Maxwell's equations and yield canonical representatives for the elements of $\mathrm{H}_{\mathrm{dR}}^{1}\left(\Sigma^{*}\right)$ and $\mathrm{H}_{\mathrm{dR}}^{1}(Y, \Sigma)$. The solution space of the $E$-cell problem is

$$
\begin{aligned}
V^{\mathrm{E}}\left(\Sigma^{*}\right) & :=\left\{\phi \in \mathrm{L}^{2}\left(Y ; \mathbb{R}^{3}\right): \operatorname{curl} \phi=0 \text { in } Y, \operatorname{div} \phi=0 \text { in } \Sigma^{*}, \phi=0 \text { in } \Sigma\right\} \\
& =\left\{\phi \in V\left(\Sigma^{*}\right): \operatorname{div} \phi=0 \text { in } \Sigma^{*}\right\} .
\end{aligned}
$$

The solution space of the $H$-cell problem is

$$
\begin{aligned}
X^{\mathrm{H}}\left(\Sigma^{*}\right) & :=\left\{u \in \mathrm{L}^{2}\left(Y ; \mathbb{R}^{3}\right): \operatorname{curl} u=0 \text { in } \Sigma^{*}, \operatorname{div} u=0 \text { in } Y, u=0 \text { in } \Sigma\right\} \\
& =\left\{u \in \mathrm{L}^{2}\left(Y ; \mathbb{R}^{3}\right):\left.u\right|_{\Sigma^{*}} \in X\left(\Sigma^{*}\right), \operatorname{div} u=0 \text { in } Y, u=0 \text { in } \Sigma\right\} .
\end{aligned}
$$

The importance of the cell problems results from the following proposition.

Proposition 4.3 (Cell problems). We consider the geometric setting outlined in Section 3. Let $\left(u^{\eta}\right)_{\eta}$ and $\left(w^{\eta}\right)_{\eta}$ be sequences in $\mathrm{L}^{2}\left(\Omega ; \mathbb{R}^{3}\right)$ that satisfy

$$
\begin{aligned}
& u^{\eta}=w^{\eta}=0 \quad \text { in } \Omega_{\eta}, \\
& \operatorname{curl} w^{\eta}=u^{\eta} \quad \text { in } \Omega \text {, } \\
& \operatorname{curl} u^{\eta}=w^{\eta} \quad \text { in } \Omega_{\eta}^{*} \text {. }
\end{aligned}
$$

We assume that $u^{\eta} \stackrel{2}{\longrightarrow} u_{0}$ and $w^{\eta} \stackrel{2}{\longrightarrow} w_{0}$ for some $u_{0}, w_{0} \in \mathrm{L}^{2}\left(\Omega \times Y ; \mathbb{R}^{3}\right)$. Then, for almost all $x \in \Omega_{i}$,

$$
u_{0}(x, \cdot) \in X^{\mathrm{H}}\left(\Sigma_{i}^{*}\right), \quad w_{0}(x, \cdot) \in V^{\mathrm{E}}\left(\Sigma_{i}^{*}\right) .
$$

For the simple proof of this proposition and for the homogenization of the timeharmonic Maxwell equations we refer to [18] and [22]. The following two lemmas show that the cell problems yield a set of representatives for the spaces $\mathrm{H}_{\mathrm{dR}}^{1}\left(\Sigma^{*}\right)$ and $\mathrm{H}_{\mathrm{dR}}^{1}(Y, \Sigma)$. 
Lemma 4.4 (E-cell problem solutions). The map

$$
V^{\mathrm{E}}\left(\Sigma^{*}\right) \rightarrow \mathrm{H}_{\mathrm{dR}}^{1}(Y, \Sigma), \quad \phi \mapsto[\phi]
$$

is an isomorphism of vector spaces.

Proof. Injectivity. Suppose that $\phi \in V^{\mathrm{E}}\left(\Sigma^{*}\right)$ and $\phi=\operatorname{grad} f$ for some $f \in \mathrm{H}^{1}(Y)$ such that $f=0$ on $\Sigma$. Then $\left.f\right|_{\Sigma^{*}} \in \mathrm{H}_{0}^{1}\left(\Sigma^{*}\right)$ and $\Delta f=\operatorname{div} \phi=0$ in $\Sigma^{*}$. Hence $f=0$ and thus $\phi=0$.

Surjectivity. Let $\phi \in \mathrm{L}^{2}\left(Y ; \mathbb{R}^{3}\right)$ such that curl $\phi=0$ in $Y$ and $\phi=0$ in $\Sigma$. Now denote by $f \in \mathrm{H}^{1}(Y)$ the solution to the boundary value problem

$$
\begin{aligned}
\Delta f & =-\operatorname{div} \phi & & \text { in } \Sigma^{*} \\
f & =0 & & \text { in } \Sigma,
\end{aligned}
$$

Then $[\phi]=[\phi+\operatorname{grad} f]$ and

$$
\begin{aligned}
& \operatorname{curl}(\phi+\operatorname{grad} f)=0 \quad \text { in } Y \\
& \operatorname{div}(\phi+\operatorname{grad} f)=0 \text { in } \Sigma^{*} \\
& \phi+\operatorname{grad} f=0 \quad \text { in } \Sigma,
\end{aligned}
$$

that is, $\phi+\operatorname{grad} f \in V^{\mathrm{E}}\left(\Sigma^{*}\right)$.

Lemma 4.5 ( $H$-cell problem solutions). The map

$$
X^{\mathrm{H}}\left(\Sigma^{*}\right) \rightarrow \mathrm{H}_{\mathrm{dR}}^{1}\left(\Sigma^{*}\right), \quad u \mapsto\left[\left.u\right|_{\Sigma^{*}}\right]
$$

is an isomorphism of vector spaces.

Proof. Injectivity. Suppose $u \in X^{\mathrm{H}}\left(\Sigma^{*}\right)$ and $\left.u\right|_{\Sigma^{*}}=\operatorname{grad} f$ for some $f \in H^{1}\left(\Sigma^{*}\right)$. As $\Delta f=0$ and the normal trace of $u$ across $\partial \Sigma^{*}$ vanishes, $f$ solves the Neumann boundary value problem

$$
\int_{\Sigma^{*}} \operatorname{grad} f \cdot \operatorname{grad} \varphi=0
$$

for all $\varphi \in \mathrm{H}^{1}\left(\Sigma^{*}\right)$. It follows that $f$ is locally constant. Hence $\left.u\right|_{\Sigma^{*}}=0$ and thus $u=0$.

Surjectivity. Let $w \in \mathrm{L}^{2}\left(\Sigma^{*} ; \mathbb{R}^{3}\right)$ be such that curl $w=0$, and let $f \in \mathrm{H}^{1}\left(\Sigma^{*} ; \mathbb{R}\right)$ with $\int_{\Sigma^{*}} f=0$ be the unique solution to the Neumann boundary value problem

$$
\int_{\Sigma^{*}} \operatorname{grad} f \cdot \operatorname{grad} \varphi=\int_{\Sigma^{*}} w \cdot \operatorname{grad} \varphi
$$

for all $\varphi \in \mathrm{H}^{1}\left(\Sigma^{*}\right)$. Set

$$
u:= \begin{cases}w-\operatorname{grad} f & \text { in } \Sigma^{*} \\ 0 & \text { in } \Sigma .\end{cases}
$$

One readily verifies that $u \in X^{\mathrm{H}}\left(\Sigma^{*}\right)$ and $[w]=\left[\left.u\right|_{\Sigma^{*}}\right]$.

\section{Maxwell's equations and effective material properties}

The most important application of the geometric average is the homogenization of Maxwell's equations in piecewise periodic geometries. In this section, we derive effective equations for general geometries which may contain inclusions of macroscopic dimension (e.g. wires). 


\subsection{Reconstructions from the geometric limit}

Proposition 5.1. We consider the geometric setting outlined at the beginning of Section 3 with the subdomains $\Omega_{1}$ and $\Omega_{2}$ and the inclusion domains $\Sigma_{1}$ and $\Sigma_{2}$. Let $\left(w^{\eta}\right)_{\eta}$ be a sequence in $\mathrm{L}^{2}\left(\Omega ; \mathbb{R}^{3}\right)$ with the two properties

$$
\begin{aligned}
w^{\eta} & =0 \text { in } \Omega_{\eta}, \\
u^{\eta} & :=\operatorname{curl} w^{\eta} \in \mathrm{L}^{2}\left(\Omega ; \mathbb{R}^{3}\right) .
\end{aligned}
$$

We assume that $u^{\eta} \stackrel{2}{\longrightarrow} u_{0}$ and $w^{\eta} \stackrel{2}{\longrightarrow} w_{0}$ for some limit functions $u_{0}, w_{0} \in \mathrm{L}^{2}\left(\Omega \times Y ; \mathbb{R}^{3}\right)$. We denote the weak limit by $\hat{w}(x):=\int_{Y} w_{0}(x, \cdot)$ for $x \in \Omega$. Then there holds, for arbitrary $v \in X\left(\Sigma_{i}^{*}\right)$,

$$
\operatorname{curl} \hat{w} \cdot \oint_{\Sigma_{i}^{*}} v=\int_{\Sigma_{i}^{*}} u_{0} \cdot v
$$

in the sense of distributions on $\Omega_{i}$.

Proof. We fix $\theta \in C_{c}^{\infty}\left(\Omega_{i}\right)$ and $v \in X\left(\Sigma_{i}^{*}\right)$. For $\eta>0$ we define $\psi(x, y):=\theta(x) v(y)$ and $\psi^{\eta}(x):=\psi(x, x / \eta)$. As $\left(u^{\eta}\right)_{\eta}$ two-scale converges to $u_{0}$, there holds

$$
\lim _{\eta \rightarrow 0} \int_{\Omega} u^{\eta}(x) \cdot \psi^{\eta}(x) \mathrm{d} x=\int_{\Omega} \theta(x)\left(\int_{Y} u_{0}(x, y) \cdot v(y) \mathrm{d} y\right) \mathrm{d} x .
$$

On the other hand, as curl $v=0$ in $\Sigma_{i}^{*}$, we find that $\operatorname{curl} \psi^{\eta}(x)=\operatorname{grad} \theta(x) \wedge v(x / \eta)$ for all $x \in \Omega_{\eta}^{*}$. Using this and (5.1a)-(5.1b), we compute

$$
\begin{aligned}
\lim _{\eta \rightarrow 0} \int_{\Omega} u^{\eta}(x) \cdot \psi^{\eta}(x) \mathrm{d} x & =\lim _{\eta \rightarrow 0} \int_{\Omega_{\eta}^{*}} \operatorname{curl} w^{\eta}(x) \cdot \psi^{\eta}(x) \mathrm{d} x \\
& =\lim _{\eta \rightarrow 0} \int_{\Omega_{\eta}^{*}} w^{\eta}(x) \cdot(\operatorname{grad} \theta(x) \wedge v(x / \eta)) \mathrm{d} x \\
& =-\int_{\Omega} \operatorname{grad} \theta(x) \cdot\left(\int_{\Sigma_{i}^{*}} w_{0}(x, y) \wedge v(y) \mathrm{d} y\right) \mathrm{d} x \\
& =-\int_{\Omega} \operatorname{grad} \theta(x) \cdot\left(\hat{w}(x) \wedge\left(\oint_{\Sigma_{i}^{*}} v\right)\right) \mathrm{d} x .
\end{aligned}
$$

Combining (5.3) and (5.4), we obtain equation (5.2).

Equation (5.2) implies that, for almost all $x \in \Omega_{i}$, the function $u_{0}(x, \cdot)$ is orthogonal to the kernel of $\mathcal{G}\left(\Sigma_{i}^{*}\right)$. In order to formalize this observation, we introduce the $L^{2}$-orthogonal decomposition

$$
X\left(\Sigma_{i}^{*}\right)=X_{0}\left(\Sigma_{i}^{*}\right) \oplus X_{1}\left(\Sigma_{i}^{*}\right) \quad \text { with } \quad X_{0}\left(\Sigma_{i}^{*}\right):=\operatorname{ker}\left(\mathcal{G}: X\left(\Sigma_{i}^{*}\right) \rightarrow A\left(\Sigma_{i}^{*}\right)^{\wedge}\right) .
$$

Corollary 5.2. In the situation of Proposition 5.1, for almost all $x \in \Omega_{i}$, there holds: If $u_{0}(x, \cdot) \in X\left(\Sigma_{i}^{*}\right)$, then $u_{0}(x, \cdot) \in X_{1}\left(\Sigma_{i}^{*}\right)$. In particular, since

$$
\mathcal{G}: X_{1}\left(\Sigma_{i}^{*}\right) \rightarrow A\left(\Sigma_{i}^{*}\right)^{\wedge \perp}
$$

is one-to-one, $u_{0}(x, \cdot)$ is uniquely determined by $\hat{u}(x):=\oint_{\Sigma_{i}^{*}} u_{0}(x, \cdot)$.

The corollary shows that the geometric average $\hat{u}(x):=\oint_{\Sigma_{i}^{*}} u_{0}(x, \cdot)$ contains all the information that is needed to reconstruct the function $u_{0}(x, y)$. We will use Corollary 5.2 in the derivation of effective equations in Theorem 5.6. 


\subsection{Effective equations}

In the following definition, we use $V^{\mathrm{E}}\left(\Sigma^{*}\right)$ from (4.1).

Definition 5.3 (Effective permittivity). Let $\Sigma \subset Y$ be an admissible microstructure. The effective permittivity is the linear map

$$
\varepsilon^{\mathrm{eff}}\left(\Sigma^{*}\right): A\left(\Sigma^{*}\right) \rightarrow A\left(\Sigma^{*}\right)
$$

that satisfies

$$
\varepsilon^{\mathrm{eff}}\left(\Sigma^{*}\right)\left(\int_{\Sigma^{*}} \phi\right) \cdot \int_{\Sigma^{*}} \phi^{\prime}=\int_{\Sigma^{*}} \phi \cdot \phi^{\prime}
$$

for all $\phi, \phi^{\prime} \in V^{\mathrm{E}}\left(\Sigma^{*}\right)$.

The effective permittivity is well-defined by (5.6). Indeed, by Lemma 4.4, the map

$$
V^{\mathrm{E}}\left(\Sigma^{*}\right) \rightarrow A\left(\Sigma^{*}\right), \quad \phi \mapsto \int_{\Sigma^{*}} \phi
$$

is an isomorphism. This implies that there is no freedom in the choice of $\phi \in V^{\mathrm{E}}\left(\Sigma^{*}\right)$ for a given average $\int_{\Sigma^{*}} \phi$, and accordingly for $\phi^{\prime}$.

Lemma 5.4. Equation (5.6) remains true if $\phi$ is chosen from the larger space $V\left(\Sigma^{*}\right)$. Similarly, also $\phi^{\prime}$ can be taken from the larger space $V\left(\Sigma^{*}\right)$ for $\phi \in V^{\mathrm{E}}\left(\Sigma^{*}\right)$.

Proof. Consider any $\psi \in V\left(\Sigma^{*}\right)$. By Lemma 4.4, there exists a function $f \in \mathrm{H}^{1}(Y)$ such that $\phi:=\psi+\operatorname{grad} f \in V^{\mathrm{E}}\left(\Sigma^{*}\right)$. But then $\int_{Y} \psi=\int_{Y} \phi$ and $\int_{Y} \psi \cdot \phi^{\prime}=\int_{Y} \phi \cdot \phi^{\prime}$ since $\operatorname{div} \phi^{\prime}=0$. This shows that (5.6) remains valid if $\phi$ is replaced by $\psi$. Analogously, one shows that (5.6) still holds when $\phi^{\prime}$ is replaced by $\psi$.

Definition 5.5 (Effective permeability). Let $\Sigma \subset Y$ be an admissible microstructure. The effective permeability is the linear map

$$
\mu^{\mathrm{eff}}\left(\Sigma^{*}\right): A\left(\Sigma^{*}\right)^{\wedge} \rightarrow \mathbb{R}^{3}
$$

defined by

$$
\mu^{\mathrm{eff}}\left(\Sigma^{*}\right)\left(\oint_{\Sigma^{*}} u\right):=\int_{\Sigma^{*}} u
$$

for all $u \in X_{1}\left(\Sigma^{*}\right)$.

The definition of $X_{1}\left(\Sigma^{*}\right)$ in (5.5) and the surjectivity of the geometric average (see Theorem 2.2) ensure that the effective permeability is well-defined by (5.7).

Theorem 5.6 (Effective equations). We consider the geometric setting outlined at the beginning of Section 3. Let $\left(u^{\eta}\right)_{\eta}$ and $\left(w^{\eta}\right)_{\eta}$ be sequences in $\mathrm{L}^{2}\left(\Omega ; \mathbb{R}^{3}\right)$ that satisfy

$$
\begin{aligned}
u^{\eta}=w^{\eta}=0 & & \text { in } \Omega_{\eta}, \\
\operatorname{curl} w^{\eta}=u^{\eta} & & \text { in } \Omega, \\
\operatorname{curl} u^{\eta}=w^{\eta} & & \text { in } \Omega_{\eta}^{*} .
\end{aligned}
$$

We assume that $u^{\eta} \stackrel{2}{\longrightarrow} u_{0}$ and $w^{\eta} \stackrel{2}{\longrightarrow} w_{0}$ for some $u_{0}, w_{0} \in \mathrm{L}^{2}\left(\Omega \times Y ; \mathbb{R}^{3}\right)$. For all $x \in \Omega_{i}$ we set

$$
\hat{u}(x):=\oint_{\Sigma_{i}^{*}} u_{0}(x, \cdot), \quad \hat{w}(x):=\int_{Y} w_{0}(x, \cdot),
$$


and

$$
\hat{\varepsilon}(x):=\varepsilon^{\mathrm{eff}}\left(\Sigma_{i}^{*}\right), \quad \hat{\mu}(x):=\mu^{\mathrm{eff}}\left(\Sigma_{i}^{*}\right)
$$

Then there holds

$$
\begin{aligned}
\hat{w}(x) & \in A\left(\Sigma_{i}^{*}\right) & & \text { for a.e. } x \in \Omega_{i}, \\
\hat{u}(x) & \in A\left(\Sigma_{i}^{*}\right)^{\wedge \perp} & & \text { for a.e. } x \in \Omega_{i}, \\
\operatorname{curl} \hat{w} & =\hat{\mu} \hat{u} & & \text { in } \Omega, \\
\pi_{A\left(\Sigma_{i}^{*}\right)}(\operatorname{curl} \hat{u}) & =\hat{\varepsilon} \hat{w} & & \text { in } \Omega_{i}, \\
\pi_{A\left(\Sigma^{*}\right)}(\operatorname{curl} \hat{u}) & =\pi_{A\left(\Sigma^{*}\right)}(\hat{\varepsilon} \hat{w}) & & \text { in } \Omega .
\end{aligned}
$$

Proof. Denote by

$$
u(x):=\int_{Y} u_{0}(x, \cdot) \quad \text { and } \quad w(x):=\int_{Y} w_{0}(x, \cdot)
$$

the weak $\mathrm{L}^{2}\left(\Omega ; \mathbb{R}^{3}\right)$-limits of $\left(u^{\eta}\right)_{\eta}$ and $\left(w^{\eta}\right)_{\eta}$, respectively.

(i) By Proposition 4.3 we know in particular that $u_{0}(x, \cdot) \in X\left(\Sigma_{i}^{*}\right)$ and $w_{0}(x, \cdot) \in V\left(\Sigma_{i}^{*}\right)$ for almost all $x \in \Omega_{i}$. Therefore, (5.11a) and (5.11b) follow from the definition of the effective fields $\hat{u}$ and $\hat{w}$ in (5.9).

(ii) It follows from (5.8b) that curl $w=u$ in $\Omega$. But $w=\hat{w}$ by definition. As for $u$, we make use of Corollary 5.2 to infer that $u_{0}(x, \cdot) \in X_{1}\left(\Sigma_{i}^{*}\right)$ for almost all $x \in \Omega_{i}$. Hence $u=\mu^{\mathrm{eff}}\left(\Sigma_{i}^{*}\right) \hat{u}=\hat{\mu} \hat{u}$ by (5.7) and (5.11c) follows.

(iii) By (3.2) and (5.6), curl $\hat{u} \cdot c=\hat{\varepsilon} \hat{w} \cdot c$ in $\Omega_{i}$ for every $c \in A\left(\Sigma_{i}^{*}\right)$. Hence (5.11d) follows.

(iv) By (3.3) and (5.6), curl $\hat{u} \cdot c=\hat{\varepsilon} \hat{w} \cdot c$ in $\Omega_{i}$ for every $c \in A\left(\Sigma^{*}\right)$. Hence (5.11e) follows.

The effective equations of Theorem 5.6 should be read as follows. In the case of compactly contained inclusions $\Sigma_{i}$, there holds $A\left(\Sigma_{1}^{*}\right)=A\left(\Sigma_{2}^{*}\right)=\mathbb{R}^{3}$ and therefore $A\left(\Sigma_{1}^{*}\right)^{\wedge \downarrow}=A\left(\Sigma_{2}^{*}\right)^{\wedge \perp}=\mathbb{R}^{3}$, see (2.4). In this case, (5.11a) and (5.11b) impose no restriction on $\hat{w}$ and $\hat{u}$ and the effective equations are curl $\hat{w}=\hat{\mu} \hat{u}$ from (5.11c) and curl $\hat{u}=\hat{\varepsilon} \hat{w}$ from $(5.11 \mathrm{e})$.

In the general case, only certain components of the curl-relations are imposed, this is expressed by the projections in (5.11d) and (5.11e). This lack of information in terms of equations is compensated by restrictions on $\hat{w}$ and $\hat{u}$ in (5.11a) and (5.11b).

The effective equations coincide with those obtained in [22].

\section{A Proof of Lemma 2.3}

Proof. We make use of the inclusion $\imath: U \rightarrow \mathbb{R}^{3}$ and the orthogonal projection $\imath^{*}: \mathbb{R}^{3} \rightarrow U$. In particular, $\imath^{*} \imath: U \rightarrow U$ is the identity. By our assumption on $B: U \rightarrow \mathbb{R}^{3}$, the linear map $\imath^{*} B: U \rightarrow U$ is skew-symmetric. We define

$$
\tilde{B}:=B \imath^{*}-\imath B^{*}-\imath \imath^{*} B \imath^{*}: \mathbb{R}^{3} \rightarrow \mathbb{R}^{3},
$$

and claim that $\tilde{B}$ is skew-symmetric. Indeed, for any $x \in \mathbb{R}^{3}$,

$$
\begin{aligned}
\tilde{B} x \cdot x & =B \imath^{*} x \cdot x-\imath B^{*} x \cdot x-\imath \imath^{*} B \imath^{*} x \cdot x \\
& =\imath^{*} x \cdot B^{*} x-B^{*} x \cdot \imath^{*} x-\imath^{*} B \imath^{*} x \cdot \imath^{*} x=0,
\end{aligned}
$$


where the last term vanishes by the skey-symmetry of $\imath^{*} B$. We claim that, for $x \in U$, there holds $\tilde{B} \imath x=B x$. Indeed, for any $y \in \mathbb{R}^{3}$,

$$
\begin{aligned}
\tilde{B} \imath x \cdot y & =B x \cdot y-\imath B^{*} \imath x \cdot y-\imath \imath^{*} B x \cdot y \\
& =B x \cdot y-x \cdot\left(\imath^{*} B\right) \imath^{*} y-\left(\imath^{*} B\right) x \cdot \imath^{*} y=B x \cdot y,
\end{aligned}
$$

since $\imath^{*} B$ is skew-symmetric. Because $\tilde{B}: \mathbb{R}^{3} \rightarrow \mathbb{R}^{3}$ is skew-symmetric, we find a (unique) vector $b \in \mathbb{R}^{3}$ such that $\tilde{B} x=b \wedge x$ for every $x \in \mathbb{R}^{3}$. In particular, $B x=b \wedge x$ for every $x \in U$.

The set of all vectors $b \in \mathbb{R}^{3}$ that represent $B$ is an affine subspace of $\mathbb{R}^{3}$, it is of the form $b_{0}+U^{\wedge}$ with the linear space

$$
U^{\wedge}=\left\{b \in \mathbb{R}^{3}: b \wedge x=0 \text { for every } x \in U\right\}= \begin{cases}\mathbb{R}^{3} & \operatorname{dim} U=0, \\ U & \operatorname{dim} U=1, \\ \{0\} & \operatorname{dim} U \geq 2 .\end{cases}
$$

In the affine space $b_{0}+U^{\wedge}$, there is a unique vector $b$ that is orthogonal to $U^{\wedge}$. The orthogonal complement of $U^{\wedge}$ is $\left(U^{\wedge}\right)^{\perp}=U^{\wedge}$, which proves the lemma.

Acknowledgement. This work was supported by the Deutsche Forschungsgemeinschaft (DFG) in the project "Wellenausbreitung in periodischen Strukturen und Mechanismen negativer Brechung", grant SCHW 639/6-1.

\section{References}

[1] G. Allaire. Homogenization and two-scale convergence. SIAM J. Math. Anal., 23(6):1482-1518, 1992.

[2] G. Bouchitté and C. Bourel. Multiscale nanorod metamaterials and realizable permittivity tensors. Commun. Comput. Phys., 11(2):489-507, 2012.

[3] G. Bouchitté, C. Bourel, and D. Felbacq. Homogenization of the 3D Maxwell system near resonances and artificial magnetism. C. R. Math. Acad. Sci. Paris, 347(9-10):571-576, 2009.

[4] G. Bouchitté, C. Bourel, and D. Felbacq. Homogenization near resonances and artificial magnetism in three dimensional dielectric metamaterials. Archive for Rational Mechanics and Analysis, 225(3):1233-1277, jun 2017.

[5] G. Bouchitté and D. Felbacq. Homogenization of a wire photonic crystal: the case of small volume fraction. SIAM J. Appl. Math., 66(6):2061-2084, 2006.

[6] G. Bouchitté and B. Schweizer. Homogenization of Maxwell's equations in a split ring geometry. Multiscale Model. Simul., 8(3):717-750, 2010.

[7] G. Bouchitté and B. Schweizer. Plasmonic waves allow perfect transmission through sub-wavelength metallic gratings. Netw. Heterog. Media, 8(4):857-878, 2013.

[8] L. Cao, Y. Zhang, W. Allegretto, and Y. Lin. Multiscale asymptotic method for Maxwell's equations in composite materials. SIAM J. Numer. Anal., 47(6):4257-4289, 2010. 
[9] X. Chen and A. Friedman. Maxwell's equations in a periodic structure. Trans. Amer. Math. Soc., 323(2):465-507, 1991.

[10] K. Cherednichenko and S. Cooper. Homogenization of the system of high-contrast Maxwell equations. Mathematika, 61(2):475-500, 2015.

[11] D. Cioranescu and J. Paulin. Homogenization in open sets with holes. J. Math. Anal. Appl., 71(2):590-607, 1979.

[12] A. Efros and A. Pokrovsky. Dielectroc photonic crystal as medium with negative electric permittivity and magnetic permeability. Solid State Communications, 129(10):643-647, 2004.

[13] D. Felbacq and G. Bouchitté. Homogenization of a set of parallel fibres. Waves Random Media, 7(2):245-256, 1997.

[14] V. V. Jikov, S. M. Kozlov, and O. A. Oleinik. Homogenization of differential operators and integral functionals. Springer-Verlag, Berlin, 1994. Translated from the Russian by G. A. Yosifian [G. A. Iosifyan].

[15] R. Kohn and S. Shipman. Magnetism and homogenization of micro-resonators. Multiscale Modeling \& Simulation, 7(1):62-92, 2007.

[16] A. Lamacz and B. Schweizer. A negative index meta-material for Maxwell's equations. SIAM J. Math. Anal., 48(6):4155-4174, 2016.

[17] A. Lamacz and B. Schweizer. Effective acoustic properties of a meta-material consisting of small Helmholtz resonators. Discrete Contin. Dyn. Syst. Ser. S, 10(4):815$835,2017$.

[18] R. Lipton and B. Schweizer. Effective Maxwell's equations for perfectly conducting split ring resonators. Archive for Rational Mechanics and Analysis, 229(3):1197-1221, Mar. 2018.

[19] G. W. Milton. Realizability of metamaterials with prescribed electric permittivity and magnetic permeability tensors. New Journal of Physics, 12(3):033035, 2010.

[20] S. O'Brien and J. Pendry. Magnetic activity at infrared frequencies in structured metallic photonic crystals. J. Phys. Condens. Mat., 14:6383-6394, 2002.

[21] J. Pendry. Negative refraction makes a perfect lens. Phys. Rev. Lett., 85(3966), 2000.

[22] B. Schweizer and M. Urban. Effective Maxwell's equations in general periodic microstructures. Applicable Analysis, 97(13):2210-2230, sep 2017.

[23] D. Smith, J. Pendry, and M. Wiltshire. Metamaterials and negative refractive index. Science, 305:788-792, 2004. 


\section{Preprints ab 2015/01}

2019-05 Klaas Hendrik Poelstra, Ben Schweizer and Maik Urban

The geometric average of curl-free fields in periodic geometries

2019-04 Agnes Lamacz and Ben Schweizer

Representation of solutions to wave equations with profile functions

2019-03 Ben Schweizer

Existence results for the Helmholtz equation in periodic wave-guides with energy methods

2019-02 Ben Schweizer and Maik Urban

On a limiting absorption principle for sesquilinear forms with an application to the Helmholtz equation in a waveguide

2019-01 Michael Voit and Jeannette H.C. Woerner

Functional central limit theorems for multivariate Bessel processes in the freezing regime

2018-08 Ben Schweizer

Effective Helmholtz problem in a domain with a Neumann sieve perforation

2018-07 Sergio Andraus and Michael Voit

Limit theorems for multivariate Bessel processes in the freezing regime

2018-06 Michael Voit

Central limit theorems for multivariate Bessel processes in the freezing regime

2018-05 Elena E1 Behi-Gornostaeva, Koondanibha Mitra and Ben Schweizer

Traveling wave solutions for the Richards equation with hysteresis

2018-04 Mario Ohlberger, Ben Schweizer, Maik Urban and Barbara Verfürth

Mathematical analysis of transmission properties of electromagnetic meta-materials

2018-03 Margit Rösler and Michael Voit

Beta distributions and Sonine integrals for Bessel functions on symmetric cones

2018-02 Michael Voit

Continuous Association Schemes and Hypergroups

2018-01 Merdan Artykov and Michael Voit

Some central limit theorems for random walks associated with hypergeometric functions of type BC

\section{7-05 Ben Schweizer and Florian Theil}

Lattice dynamics on large time scales and dispersive effective equations

2017-04 Frank Klinker and Christoph Reineke

A note on the regularity of matrices with uniform polynomial entries

2017-03 Tomáš Dohnal and Ben Schweizer

A Bloch wave numerical scheme for scattering problems in periodic wave-guides

2017-02 Matthias Röger and Ben Schweizer

Strain gradient visco-plasticity with dislocation densities contributing to the energy

2017-01 Ben Schweizer and Maik Urban

Effective Maxwell's equations in general periodic microstructures 
2016-04 Ben Schweizer

Resonance meets homogenization - Construction of meta-materials with astonishing properties

2016-03 Ben Schweizer

On Friedrichs inequality, Helmholtz decomposition, vector potentials, and the div-curl lemma

2016-02 Michael Voit

Generalized commutative association schemes, hypergroups, and positive product formulas

2016-01 Agnes Lamacz and Ben Schweizer

Effective acoustic properties of a meta-material consisting of small Helmholtz resonators

Christian Eggert, Ralf Gäer, Frank Klinker

The general treatment of non-symmetric, non-balanced star circuits: On the geometrization of problems in electrical metrology

2015-12 Daniel Kobe and Jeannette H.C. Woerner

Oscillating Ornstein-Uhlenbeck processes and modelling electricity prices

2015-11 Sven Glaser

A distributional limit theorem for the realized power variation of linear fractional stable motions

2015-10 Herold Dehling, Brice Franke and Jeannette H.C. Woerner

Estimating drift parameters in a fractional Ornstein Uhlenbeck process with periodic mean

2015-09 Harald Garcke, Johannes Kampmann, Andreas Rätz and Matthias Röger

A coupled surface-Cahn-Hilliard bulk-diffusion system modeling lipid raft formation in cell membrans

2015-08 Agnes Lamacz and Ben Schweizer

Outgoing wave conditions in photonic crystals and transmission properties at interfaces

2015-07 Manh Hong Duong, Agnes Lamacz, Mark A. Peletier and Upanshu Sharma

Variational approach to coarse-graining of generalized gradient flows

2015-06 Agnes Lamacz and Ben Schweizer

A negative index meta-material for Maxwell's equations

2015-05 Michael Voit

Dispersion and limit theorems for random walks associated with hypergeometric functions of type $B C$

2015-04 Andreas Rätz

Diffuse-interface approximations of osmosis free boundary problems

2015-03 Margit Rösler and Michael Voit

A multivariate version of the disk convolution

2015-02 Christina Dörlemann, Martin Heida, Ben Schweizer

Transmission conditions for the Helmholtz-equation in perforated domains

2015-01 Frank Klinker

Program of the International Conference

Geometric and Algebraic Methods in Mathematical Physics

March 16-19, 2015, Dortmund 